\title{
On the Search for the Perfect Mismatch!
}

$\mathbf{T}$ he selection of candidates who are likely to benefit from reperfusion therapy has been a focus of research in acute ischemic stroke. This produced a number of paradigms to the conceptual approach and imaging of patients with acute ischemic stroke. These paradigms aim to establish the presence of a sizable salvageable brain tissue that justifies taking the risks of medical interventions to achieve reperfusion. This introduced the concept of mismatch: a clinically important difference between the brain tissue that is already infarcted (the core) and the tissue that is on its way to infarct if complete reperfusion is not rapidly achieved (the penumbra). The identification of mismatch has been a premise of acute stroke imaging either to allow the treatment of those with an onset time that is unknown or outside the conventional time window; or to help select candidates for endovascular therapies after intravenous thrombolysis.

The evidence to support improved functional outcomes with the use of a mismatch paradigm is yet to be shown in randomized clinical trials. Trials of intravenous thrombolysis ${ }^{1}$ or endovascular therapies ${ }^{2}$ have failed to show a significant difference in clinical outcomes between patients with acute stroke selected based on the detection of mismatch versus those selected based on typical clinical or imaging criteria. An important challenge is the dynamic and heterogeneous nature of ischemic stroke contrary to the static quality of many of the available imaging modalities. This makes the a priori prediction of tissue fate often flawed, especially when rapid reperfusion is increasingly achieved using contemporary endovascular devices. In addition, the distinction between the core versus the penumbra and the penumbra versus areas of benign oligemia may not be as well delineated as what is perceived by viewing the color-coded perfusion maps. ${ }^{3}$

An emerging paradigm that has received special attention in recent years is the assessment of the collateral circulation. These vascular channels are believed to sustain the penumbra for a relatively longer period of time and their extent is thought to be directly proportional to the volume of the penumbra. Patients with good collaterals have fared clinically better after thrombolytic or endovascular therapies compared with patients with poor collaterals even when reperfusion was achieved. ${ }^{4-6}$ Traditionally, the collateral status was assessed in the acute stroke setting using conventional angiography. ${ }^{7}$ However, the advancement of noninvasive imaging techniques provided more readily available alternatives. Single-phase CTA assessment of leptomeningeal collaterals produced a number of scores, all of which concluded that better collaterals were associated with smaller infarct volume or better functional outcome than poor collaterals. ${ }^{8-10}$ The sensitivity of single-phase CTA to the timing of image acquisition in relation to the bolus led researchers to develop techniques to provide dynamic collateral assessment. This produced time-resolved, CT-based collateral imaging techniques that used partial ${ }^{11}$ or complete $^{12}$ brain coverage to assess the temporal filling of collaterals beyond an arterial occlusion. These data provided grounds for excluding patients with poor or no collaterals on multiphase $\mathrm{CTA}^{12}$ from the ongoing Endovascular treatment for Small Core and Anterior circulation Proximal occlusion with Emphasis on minimizing CT to recanalization times (ESCAPE) trial.

The role of MR imaging in collateral assessment relied on few techniques. ${ }^{13}$ The presence of distal hyperintense vessels on FLAIR imaging, ${ }^{14}$ believed to reflect good collateral status, corresponded to smaller core volume, favorable diffusion-perfusion mismatch, and milder stroke severity. Collateral assessment using arterial spin-labeling has shown good agreement with conventional angiography in patients with cervical or intracranial stenosis in the nonacute setting. ${ }^{15}$ Scattered reports described the potential utility of susceptibility-weighted imaging techniques in this context. ${ }^{13,16}$ It is hypothesized that the increased brain tissue demand for oxygen downstream from an occlusion leads to a higher concentration of deoxyhemoglobin in the capillaries and venous outflow channels leading to a susceptibility difference that discriminates these vessels from the adjacent brain. ${ }^{17}$ The detection of these channels is thought to signify the presence of penumbra.

In this issue of the American Journal of Neuroradiology, a mismatch paradigm based on the venous outflow estimation is used to define a susceptibility/diffusion (SWI/DWI) mismatch. This previously described paradigm ${ }^{18-20}$ is objectively measured to assess the relationship between clinical outcome and an SWI/DWI mismatch in 54 patients with stroke treated with IV tPA. Automated venous SWI segmented maps were generated and com- 
pared with the contralateral side to calculate an asymmetry index. Patients with more than 1.75 asymmetry index and DWI volume of $\leq 25 \mathrm{~mL}$ achieved over $90 \%$ favorable outcome with recanalization. The SWI/DWI mismatch was a better predictor of functional independence than the PWI/DWI mismatch in this study.

Despite a number of limitations, many of which were acknowledged by the investigators, the study findings may have important future applications. Once the method to identify this SWI/DWI mismatch is simplified and accessible, studying this paradigm in past or ongoing large prospective cohorts will help advance the understanding of its significance and define its role in patient selection for reperfusion therapies outside the standard window or via the endovascular approach. Another interesting aspect will be to investigate any regional correlation of these venous outflow channels with penumbral tissue or the presence of robust collaterals. Once its validity is established, this imaging paradigm will face the practical challenges that all MR-based acute stroke techniques need to overcome ${ }^{21}$ in addition to the lack of evidence from a randomized trial supporting the improved outcomes of patients selected using penumbral imaging. ${ }^{2}$

Whether it is collateral-, tissue-, or venous outflow-based mismatch, the important question remains: which of these paradigms will be shown to predict improved functional outcomes? To achieve this, it will also need to be readily available, easily interpretable with minimal postprocessing, and able to be efficiently incorporated into the hyperacute stroke workflow to allow timely reperfusion. Finding the "perfect" mismatch is not easy after all!

\section{REFERENCES}

1. Davis SM, Donnan GA, Parsons MW, et al. Effects of alteplase beyond $3 \mathrm{~h}$ after stroke in the Echoplanar Imaging Thrombolytic Evaluation Trial (EPITHET): a placebo-controlled randomised trial. Lancet Neurol 2008;7:299-309

2. Kidwell CS, Jahan R, Gornbein J, et al. A trial of imaging selection and endovascular treatment for ischemic stroke. $N$ Engl J Med 2013;368:914-23

3. Kidwell CS, Alger JR, Saver JL. Beyond mismatch: evolving paradigms in imaging the ischemic penumbra with multimodal magnetic resonance imaging. Stroke 2003;34:2729-35

4. Bang OY, Saver JL, Buck BH, et al. Impact of collateral flow on tissue fate in acute ischaemic stroke. J Neurol Neurosurg Psychiatry 2008;79:625-29

5. Nambiar V, Sohn SI, Almekhlafi MA, et al. CTA collateral status and response to recanalization in patients with acute ischemic stroke. AJNR Am J Neuroradiol 2014;35:884-90

6. Liebeskind DS, Tomsick TA, Foster LD, et al. Collaterals at angiog- raphy and outcomes in the Interventional Management of Stroke (IMS) III trial. Stroke 2014;45:759-64

7. Bozzao L, Fantozzi LM, Bastianello S, et al. Early collateral blood supply and late parenchymal brain damage in patients with middle cerebral artery occlusion. Stroke 1989;20:735-40

8. Maas MB, Lev MH, Ay H, et al. Collateral vessels on CT angiography predict outcome in acute ischemic stroke. Stroke 2009;40:3001-05

9. Menon BK, O'Brien B, Bivard A, et al. Assessment of leptomeningeal collaterals using dynamic $C T$ angiography in patients with acute ischemic stroke. J Cereb Blood Flow Metab 2013;33:365-71

10. Miteff F, Levi CR, Bateman GA, et al. The independent predictive utility of computed tomography angiographic collateral status in acute ischaemic stroke. Brain 2009;132:2231-38

11. Frolich AM, Wolff SL, Psychogios MN, et al. Time-resolved assessment of collateral flow using 4D CT angiography in large-vessel occlusion stroke. Eur Radiol 2014;24:390-96

12. Goyal M, Menon B. Optimizing acute stroke imaging for maximizing information and minimizing acquisition, post processing and interpretation times: analysis of data from a prospective imaging cohort study. In: Proceedings of the European Stroke Conference, London, UK. May 28-31, 2013

13. McVerry F, Liebeskind DS, Muir KW. Systematic review of methods for assessing leptomeningeal collateral flow. AJNR Am J Neuroradiol 2012;33:576-82

14. Lee KY, Latour LL, Luby M, et al. Distal hyperintense vessels on FLAIR: an MRI marker for collateral circulation in acute stroke? Neurology 2009;72:1134-39

15. Wu B, Wang X, Guo J, et al. Collateral circulation imaging: MR perfusion territory arterial spin-labeling at $3 \mathrm{~T}$. AJNR Am J Neuroradiol 2008;29:1855-60

16. Hermier M, Nighoghossian N, Derex L, et al. Hypointense leptomeningeal vessels at T2*-weighted MRI in acute ischemic stroke. Neurology 2005;65:652-53

17. Hermier M, Nighoghossian N. Contribution of susceptibilityweighted imaging to acute stroke assessment. Stroke 2004;35: 1989-94

18. Kao HW, Tsai FY, Hasso AN. Predicting stroke evolution: comparison of susceptibility-weighted MR imaging with MR perfusion. Eur Radiol 2012;22:1397-403

19. Meoded A, Poretti A, Benson JE, et al. Evaluation of the ischemic penumbra focusing on the venous drainage: the role of susceptibility weighted imaging (SWI) in pediatric ischemic cerebral stroke. J Neuroradiol 2014;41:108-16

20. Fujioka M, Okuchi K, Iwamura A, et al. A mismatch between the abnormalities in diffusion- and susceptibility-weighted magnetic resonance imaging may represent an acute ischemic penumbra with misery perfusion. J Stroke Cerebrovasc Dis 2013;22:1428-31

21. Wisco D, Uchino K, Saqqur M, et al. Addition of hyperacute MRI aids in patient selection, decreasing the use of endovascular stroke therapy. Stroke 2014;45:467-72

M.A. Almekhlafi

King Abdulaziz University Jeddah, Saudi Arabia

http://dx.doi.org/10.3174/ajnr.A4024 